http://dx.doi.org/10.18778/2196-8403.2018.04

\title{
Zu Bildungsverfahren und Strukturen von Benennungsausdrücken. Eine Analyse anhand von Bezeichnungen für die staatspolitischen Veränderungen 1989
}

Der Beitrag stellt den Begriff der Benennung vor und geht auf Probleme der Zwecke / Motive des Benennens ein. Anhand von Pressebeispielen aus einem bestimmten gesellschaftlich-politischen Bereich wird eine Übersicht über Bildungsverfahren und Strukturen von Benennungseinheiten gegeben. Die sprachliche Analyse konzentriert sich im Detail auf wortbildende, syntaktische und semantische Verfahren, die dazu verwendet wurden, Objekte zu benennen.

Methods of word formation and structures in the process of nomination. A case study based on lexical units from the socio-political field of 1989

The paper begins by defining the concept of nomination and then discusses the reasons and motives in the process of nomination. Based on corpus-driven examples from social and political news stories, the article presents an overview of methods of word formation as well as various types of lexical nomination units. The linguistic analysis focuses on word formation mechanisms, syntactic and semantic methods used in the process of nomination of objects.

O sposobach tworzenia i strukturach jednostek nominacyjnych. Analiza na przykładzie określeń dotyczących przemian społeczno-politycznych roku 1989

W artykule przedstawiono pojęcie nominacji oraz poruszono kwestie celów / motywów nadawania nazw. Na przykładach prasowych dotyczących konkretnych wydarzeń społeczno-politycznych zaprezentowano przegląd sposobów tworzenia nazw oraz przedstawiono szereg struktur jednostek nominacyjnych. Analiza językowa koncentruje się na mechanizmach słowotwórczych, syntaktycznych i semantycznych, które zostały wykorzystane do nadawania obiektom nazw. 


\section{Vorbemerkungen}

Den Ausgangspunkt der folgenden, empirisch ausgerichteten linguistischen Überlegungen bildet die Benennungsfunktion des Wortes. In den einschlägigen lexikografischen und lexikologischen Arbeiten werden neben Benennung / Benennen auch die Termini Namengebung und Nomination verwendet (vgl. FLEISCHER 1989, SCHIPPAN 1992, KNOBLOCH / SCHAEDER 1996, KNOBLOCH 1996, FEINE 1997; 1999; 2000, GŁADYSZ 2006; 2007). Benennungen werden auch als Resultat des Benennungsprozesses (so z. B. in KNOBLOCH / SCHAEDER 1996:7), also als gebildete Bezeichnungen, sprachliche Strukturen oder Ausdrücke aufgefasst. Im Rahmen dieses Beitrags sollen (in Anlehnung u. a. an KNOBLOCH / SCHAEDER 1996:7 und FEINE 2000:12) folgende terminologische und definitorische Regelungen getroffen werden:

- Benennung / Benennen ist eine sprachliche Handlung, über die (a) durch Zuordnung neuer sprachlicher Einheiten dem Konzept eines Gegenstandes oder dem Sachverhalt als kognitiv-konzeptueller Struktur (Proposition) eine Benennung zugeschrieben wird oder diese (b) durch Verwendung bereits identifizierter, ausgewählter Einheiten in Bezug auf neue Konzeptualisierungen der Gegenstände und Sachverhalte Rezipierenden verfügbar gemacht wird.

- Mit Gegenständen und Sachverhalten meine ich hier die materiellen Elemente der außersprachlichen, äußeren Kommunikationssituation wie Ort, Gegenstände am Ort, Ereignisse, Handlungen, Geschehensabläufe, die daran beteiligten Personen, Eigenschaften usw., die benannt werden.

- Das Resultat der Benennung / des Benennens (als sprachlicher Handlung) ist die gebildete oder ausgewählte sprachliche Einheit (Bezeichnung); von der grammatischen Ebene her betrachtet sind es morphosyntaktische Einheiten (Wörter / Wortformen und Wortgruppen).

\section{Zielsetzung}

Das Ziel dieses Beitrags ist zum einen die Identifikation und Beschreibung der Bezeichnungen auf der grammatischen Ebene, die die staatspolitischen Veränderungen von 1989 als thematischen Schwerpunkt haben; sie werden als wortfamiliär strukturierte Felder anhand der in den Herbstmonaten des Jahres 1989 herausgegebenen Presseberichte und Kommentare zusammengestellt. Zum an- 
deren werden diese Bezeichnungen als Indikatoren der sprachlichen Ausgestaltung der Wirklichkeit in Zeiten politischen und gesellschaftlichen Umbruchs interpretiert. Bereits an dieser Stelle wird es notwendig, Benennen als sprachliche Handlung zu präzisieren bzw. einzuordnen. Sprachliche Handlungen dienen der Kommunikation ${ }^{1}$. Das Benennen stellt für den hier relevanten Zusammenhang nur dann einen Fall von Kommunikation dar, wenn (a) es in der Absicht vollzogen wird, dem anderen etwas zu erkennen zu geben und (b) dieser auf Basis seines Situations- oder seines allgemeinen Weltwissens erkennen kann, wozu man ihn bringen möchte. In dieser Hinsicht ist anzunehmen, dass Sprechende auf Elemente unseres Wissens (d. h. die sie als eigenständige Objekte, Gegenstände, Sachverhalte usw. der wahrgenommenen außersprachlichen Welt zugelassen haben) ${ }^{2}$ mittels sprachlicher Äußerungen (explizit oder implizit) verweisen können; wie POLENZ (1985:116-143) hervorgehoben hat, führen sie dann die sprachlich-kommunikative (Teil-)Handlung des „Bezugnehmens/Referierens“" aus. An dieser Stelle ist die Annahme hinreichend, dass die Kommunikationsbeteiligten in einer bestimmten Kommunikationssituation wissen, auf welche Wissenselemente ein sprachlicher Ausdruck in dieser (und nur in dieser) konkreten Konstellation aktuell verweist (zum verstehensrelevanten Wissen vgl. ausführlich BUSSE 1992). Hier soll folgende informelle Darstellung der Zwecke oder Motive des Benennens vorgeschlagen werden, die als Faktor beim Benennungsprozess (nicht als Faktor beim (Text-)Verstehen) konstitutiv sind; sie sind u. a. funktional bestimmt (vgl. BUSSE 1992:158; KELLER 1995:17-21, 113f.; FEINE 2000:12f.; WAGNER 2001:Teil II; GŁADYSZ 2006:105; GOŁĘBIOWSKI / ENGEL 2014:68):

(1) Die Sprechenden benennen Gegenstände und Sachverhalte zur Identifizierung durch die Rezipierenden; es sind referierende Ausdrücke. Es kann nicht immer gesagt werden, ob die Kommunikationsbeteiligten mit

1 Ich verwende dieses Wort im in der Linguistik und Sprachphilosophie üblichen Sinn, nach dem Kommunikation - aus der Perspektive der Sprechenden gesehen - jedes intentionale Verhalten bedeutet und Interpretationsbemühungen der kommunikativen Intentionen (Perspektive der Interpretierenden) berücksichtigt. Hier sind nur einige wichtige Arbeiten zu nennen: GRICE (1957/1979), GRICE (1969 / 1979), Posner (1992: Kap. 3.3.), Keller (1995: Teil II, Teil III).

2 Andere Wissenselemente, die diesen erkenntnistheoretischen Status nicht haben, aber für die hier gemeinte Referenz- und Benennungshandlung Voraussetzung sind, werden psycholinguistisch oder kognitionspsychologisch als Begriffe oder Konzepte bezeichnet (so in der Psycholinguistik oder KI-Forschung). 
dieser Bezeichnung denselben Sinn verbinden (eigene oder gesellschaftliche Erfahrungen, Einstellungen usw. als Faktoren beim Textverstehen werden hier nicht weiter diskutiert). Es kommt in diesem Zusammenhang auf die Referenzfixierung an. Innerhalb der Referenzfunktion kann man (z. B. mit BUSSE 1992:155) zwei Typen von Referenz unterscheiden: Eine textinterne, für die Herstellung von Textkohärenz wesentliche Referenz und die textexterne Referenz, also die Bezugnahme auf textexterne Wissenselemente (d. i. die außersprachliche ,Welt ${ }^{\star}$ ).

(2) Die Sprechenden charakterisieren Gegenstände und Sachverhalte, indem sie bestimmte Merkmale hervorheben: den benannten Objekten werden Eigenschaften zugewiesen, die benannten Objekte werden mittels ausgewählter Bezeichnungen zu anderen in Beziehung gesetzt, die benannten Objekte werden nach Anzahl, Größe, Umfang spezifiziert; die Eigenschaften der benannten Objekte können auch verstärkt, abgeschwächt oder negiert werden. Die Rede ist von einzelnen Sprachelementen, von denen alle Substantive prototypisch für die referenzielle Funktion sind.

(3) Die Sprechenden indizieren durch das Benennen ihre Einstellung zu Gegenständen und Sachverhalten und auch deren Bewertung. Ein Idealfall liegt vor, wenn die Rezipierenden die Bedeutung der Wörter samt ihrer Verknüpfung kennen, die die Sprechenden einsetzen, bzw. sie erschließen können. Hier ist Raum für kreative Möglichkeiten seitens der Sprechenden bzw. Schreibenden, und zwar mittels neuer, okkasioneller Bezeichnungen, die keine festgeprägten Standardbezeichnungen sind, eine individuelle Stellungnahme zu einem Sachverhalt mitzuteilen. Neben der kreativsprachspielerischen Motivation werden neue Bezeichnungen auch aus dem (individuellen) Bedürfnis nach Schließung einer lexikalischen Lücke oder aus sprachökonomischen Gründen heraus geschaffen. Die letztgenannte Motivation führt dazu, dass in relativ einfachen, kurzen Strukturen Informationen, Meinungskundgaben und emotionale Stellungnahmen der Sprechenden zu komplexen Sachverhalten zusammengefasst werden können.

(4) Die Sprechenden wollen durch das Benennen bestimmte Reaktionen bei den Rezipierenden auslösen, auf ihr Verhalten einwirken. Aus der Perspektive der Sprechenden gesehen dienen ihnen gewählte Strukturen dazu, den anderen zu erkennen zu geben, wozu sie sie bringen möchten, in der Hoffnung, dass die Rezipierenden dies erkennen werden und sich in der gewünschten Weise beeinflussen lassen (z. B. eine erwünschte Einstellung zu bestimmten Sachverhalten übernehmen). Das beabsichtige Beeinflussungsziel muss selbstverständlich keinen negativen Hintergrund haben, 
es können genauso positive Absichten dahinterstecken, wie zum Beispiel sprachspielerische Zwecke, Freude bereiten etc.

(5) Die Sprechenden benennen schließlich Gegenstände und Sachverhalte auch zur Selbstidentifizierung (Identifizierung mit einer Gruppe, mit einer bestimmten gesellschaftlichen Schicht, Nation, Kultur) und Selbstdarstellung (z. B. durch Darstellung von Sozialprestige, Wohlstand oder Bildung, also von Werten, die man hat oder gerne für sich beanspruchen würde; sie verwenden hierfür sprachliche Mittel, deren Entschlüsselung Kennerschaft voraussetzt).

Zur Grundlage der vorliegenden Analyse wird ein Faktum gewählt: Das Resultat der Benennung ist immer eine sprachliche Einheit (Bezeichnung, sprachliche Struktur, Nominationseinheit, Ausdruck), die dem Konzept eines Gegenstandes oder Sachverhalts zugeordnet wird. Die sprachlichen Einheiten können mit Hilfe des morphosyntaktischen Verfahrens gebildet werden. Dessen Effekt sind Ableitungen, Zusammensetzungen, Kurzwörter, Wortgruppen; den nominalen Einheiten mit prototypisch referenzieller Funktion können Attribute beigefügt werden. Eine referenzielle Funktion im weiteren Sinne können auch Adverbien und Partikeln haben, die auf Einstellungen der Sprechenden verweisen. Die Identifikation und Beschreibung der Ausdrücke auf der grammatischen Ebene bilden das oberste Ziel in diesem Beitrag. Im zweiten Schritt will ich ausführen, dass das von Sprechenden gewählte Benennungsverfahren in einem Zusammenhang mit den Benennungszwecken und Benennungsmotiven steht, die in einer sprachlichen Einheit (Wort / Wortform, Wortgruppe) zum Ausdruck gebracht werden.

\section{Zum Belegkorpus und zur Quellenauswahl}

Um dem übergeordneten Thema des Bandes Rechnung zu tragen, ist die Auswahl des Textkorpus von der Absicht geleitet, Benennungen aus dem politischgesellschaftlichen Themenfeld zu analysieren. Das Belegkorpus bilden Benennungen zu den politischen und gesellschaftlichen Umbrüchen des Jahres 1989 in Deutschland. Für die Analyse werden die Benennungsausdrücke zu fünf Objekten (Wende, Wiedervereinigung, BRD, DDR, deutsche Bürger) aus meinungsbildenden Texten (u. a. aus Nachrichten, Kommentaren und Leserbriefen) der Berliner tageszeitung (taz) aus den Herbstmonaten des Jahres 1989 
berücksichtigt. ${ }^{3}$ Das Belegkorpus zählt fast 500 Ausdrücke. Aus Platzgründen muss es reichen, im vorliegenden Artikel für jedes analysierte Bildungsverfahren ausgewählte Beispiele zu zitieren. Bereits in der Zusammenschau der Bildungsverfahren wird deutlich, welche Auswirkungen politische und gesellschaftliche Veränderungen auf die Sprache im Sinne der Aktivierung ihrer konkreten Strukturen hatten.

Als Belegquelle werden Zeitungstexte gewählt. Sie eigenen sich sehr gut für diese Analyse, denn eine favorisierte Aufgabe der Presse (wie auch anderer Medien) ist die Vermittlung einer möglichen Wirklichkeit. Den taz-Texten werden alle Benennungsausdrücke entnommen, die in oben genannten Objektbereichen vorkommen, unabhängig davon, ob die sprachliche Vermittlungsweise im konkreten Kontext und Text sachbetont oder meinungsbetont ist. Die sachbetonte Vermittlungsweise kann sich auch mit einer meinungsbildenden, zudem mit einer evaluativen Einstellung verbinden (zu Textfunktionen vgl. LÜGER 1995:17; BRINKER 2005:113-130), und klare Grenzen sind nicht immer einfach zu umreißen. So wird im weiteren Schritt versucht zu kommentieren, ob hinter den Benennungen objektive Informationswiedergabe, spezifische Bewertungen oder Emotionen stehen.

\section{Bildungsverfahren: eine Übersicht}

Im Folgenden werden sprachliche Bildungsverfahren dargestellt, die dazu verwendet wurden, Objekte zu benennen. Die Objekte des Benennens können wie alle Wissenselemente der Wissenswelt (nach der oben beschriebenen Auffassung) eines Individuums sein: Personen, Gegenstände, Sachverhalte, Handlungen, Eigenschaften usw., in semantischer Redeweise: Konkreta, Abstrakta, Eigennamen usw. Es macht wenig Sinn, eine solche Differenzierung vornehmen zu wollen. Innerhalb der Beschreibung der Bildungstypen ist wichtig, welche Sprachelemente die Funktion des Benennens haben. Dieses ruht auf der Relation zwischen der sprachlichen Ausdrucksseite und einem benannten Wissens-

3 Mittlerweile gibt es unzählige linguistische Untersuchungen und Veröffentlichungen zu den Schlüsselwörtern dieser Umbruchsphase, in denen Sprachwissenschaftlerinnen und Sprachwissenschaftler nicht nur häufig verwendete Wörter auflisten, sondern auch ausführliche Interpretationen, Erklärungen von Wortveränderungen und eine zeitliche und thematische Eingruppierung der Wende-Wörter liefern, vgl. z. B. Herberg / StefFens / Tellenbach (1997) oder Hellmann (2006). 
ausschnitt auf. Alle Substantive (bzw. Nomen), die traditionell als Autosemantika bezeichnet werden, haben referenzielle Funktion und wurden am häufigsten für das Benennen verwendet. Auch alle Sprachelemente, die nicht allein syntaktische Bedeutung haben, weisen referenzielle (als zu einer syntaktischen Bedeutung hinzutretende) Bedeutung auf (z. B. Attribute). Ebenso haben die Wortbildungsmorpheme referenzielle Bedeutung. So handelt es sich um wortbildende, syntaktische und semantische Verfahren, die oft miteinander kombiniert auftreten. Sie werden in der Übersicht jeweils durch konkrete Beispiele gestützt. Bei den Beispielen werden die genauen Zitierangaben in Klammern entsprechend markiert. Der Übersicht folgt ein Kommentar zu den jeweiligen Benennungsmotiven.

Benennungsobjekt 1: Wende, Wendezeit (als Umbruchphase und Annäherungszeit beider deutscher Staaten)

(a) Wortbildendes Verfahren und wortbildend-semantisches Verfahren

- Zusammensetzungen (Wende als Grundwort): die Krenz-Wende (taz 01.11.89, S. 14), 360-Grad-Wende? (taz 06.11.89, S. 2), Trendwende? (taz 15.11.89, S. 15)

- Zusammensetzungen, bei denen als Grundwort oder Bestimmungswort ein Nomen steht, mit dem politische Wende metaphorisch aufgefasst wird: ${ }^{4}$ das SED-,, Geplapper “ (taz 18.10.89, S. 1), „Dialog “Geschwafel (taz 18.10.89, S. 1), ,Stunde-Null“-Rhetorik (taz 13.11.89, S. 7), die „November-Revolution“ ( $\operatorname{taz} 20.11 .89$, S. 5)

\section{(b) Syntaktisches Verfahren und syntaktisch-semantisches Verfahren}

- Wortgruppe (adjektivisches Attribut +Wende): die prokapitalistische Wende (taz 20.11.89, S. 5)

- Wortgruppen (adjektivisches Attribut + Nomen, das metaphorisch oder metonymisch für Wende(zeit) steht): der derzeitige ,, nationale Taumel“ (taz 03.10.89, S. 4), eine ,rhetorische Revolutionsromantik" (taz 08.11.89, S. 5), friedliche und demokratische Revolution (taz 11.11.89, S. 2), eine kluge und sanfte Revolution (taz 11.11.89, S. 4),

Alle Belege, die den metaphorischen oder metonymischen Gebrauch darstellen, können natürlich als solche nur im breiteren taz-Kontext ersichtlich gemacht werden. Aus Platzgründen soll jedoch auf das Zitieren längerer Passagen verzichtet werden. 
eine friedliche, demokratische Revolution (taz 11.11.89, S. 5), die Ostberliner Revolution (taz 14.11.89, S. 4), die zweite Deutsche Novemberrevolution (taz 16.11.89, S. 12-13), eine bürgerlich-demokratische Revolution (taz 16.11.89, S. 18), eine demokratisch-politische Revolution (taz 17.11.89, S. 8), Deutscher November (taz 18.11.89, S. 25-26), die erste erfolgreiche deutsche Revolution (taz 24.11.89, S. 11)

- Wortgruppen (adjektivisches Attribut + Nomen, das metaphorisch oder metonymisch für Wende(zeit) steht + Genitivattribut / präpositives Attribut/ situatives Attribut): das große Papperlapapp des Volkes (taz 09.11.89, S. 9), die friedliche Revolution vom November 1989 (taz 11.11.89, S. 12), die Novemberrevolution des Jahres 1989 in der Deutschen Demokratischen Republik (taz 16.11.89, S. 12-13), die gewaltfreie Revolution der Freiheit und Demokratie in der DDR (taz 16.11.89, S. 12-13), die "friedliche Revolution" in der DDR (taz 27.11.89, S. 4)

- Wortgruppen (ein Nomen für Wende(zeit) + Genitivattribut): eine Wiedergeburt des Pan-Germanismus (taz 16.11.89, S. 8)

\section{(c) Semantisches Verfahren ${ }^{5}$}

- Anspielungen auf das Ereignis der Oktoberrevolution von 1917 in Russland (vom 25. Oktober [julianischer Kalender] / 7. November [gregorianischer Kalender]) ${ }^{6}$; es entstehen Wortgruppen, in denen der nominale Kern entsprechend situationsaktuell attribuiert wird: die Oktoberrevolution von 1989 (taz 06.11.89, S. 1), die 1989er Oktoberrevolution (taz 06.11.89, S. 2), [jetzt, 1989, haben wir] unsere Oktoberrevolution (taz 20.11.89, S. 6)

Mit semantischem Verfahren meine ich ein selbständiges Segment, obwohl sie (die Semantik bzw. die Konstitution von Bedeutung) ja natürlich dasjenige ist, dem alle sprachlichen Mittel dienen. Im Begriff der referenziellen Funktion sind alle sprachlichen Verfahren enthalten, die die Beziehbarkeit von Sprachelementen auf Objekte der realen Welt realisieren, dies aber in unterschiedlichem Verhältnis.

Als sprachlich-kreative Anspielungen auf dieses Geschehen sind auch obige Beispiele mit dem nominalen Kern Novemberrevolution und Revolution anzusehen. 
Benennungsobjekt 2: Wiedervereinigung (als Prozess und bezogen auf den Tag der Wiedervereinigung); inkl. Bezeichnungen für die Wiedervereinigungsstimmungen

(a) Wortbildendes Verfahren und wortbildend-syntaktisches Verfahren

- Zusammensetzungen mit lexikalischem Austausch im Erstglied des Nomens (mit semantischem Effekt): Neuvereinigung (taz 01.12.89, S. 19), Niedervereinigung (taz 01.12.89, S. 19)

- Zusammensetzungen mit dem Nomen Wiedervereinigung bzw. (vereinzelt) Vereinigung als Bestimmungswort, häufig in Kombination mit syntaktischem Verfahren (Attribuierungen): die Wiedervereinigungsrhetorik (taz 02.10.89, S. 3), Wiedervereinigungsgeschrei (taz 02.10.89, S. 9), die immer wiederaufflammende ,Wiedervereinigungsrhetorik" (taz 05.10.89, S. 22), Wiedervereinigungsgedröhn (taz 07.10.89, S. 4), Wiedervereinigungsfloskeln von vorgestern (taz 12.10.89, S. 17), Wiedervereinigungsromantik (taz 26.10.89, S. 8), Wiedervereinigungsrufe (taz 01.11.89, S. 5), der Vereinigungsrausch (taz 13.11.89, S. 4), die Kohlschen Wiedervereinigungshoffnungen (taz 13.11.89, S. 8), Wiedervereinigungsrhetorik (taz 14.11.89, S. 2), Vereinigungsgedanken (taz 14.11.89, S. 3), Wiedervereinigungsgerede (taz 14.11.89, S. 20), Wiedervereinigungssog (taz 15.11.89, S. 2), großdeutsche Wiedervereinigungsszenarien (taz 16.11.89, S. 22), Wiedervereinigungsphantasien (taz 17.11.89, S. 8), Wiedervereinigungswünsche (taz 18.11.89, S. 9), die Wiedervereinigungsdrohung (taz 18.11.89, S. 9), der nationale Wiedervereinigungswahn (taz 21.11.89, S. 12), ,Wiedervereinigungseuphorie“ (taz 06.12.89, S. 16), die Wiedervereinigungsfanfare Kohls (taz 12.12.89, S. 8), Bonner Wiedervereinigungsgelüste (taz 18.12.89, S. 5)

\section{(b) Syntaktisches Verfahren und syntaktisch-semantisches Verfahren}

- Wortgruppen (adjektivisches Attribut + Wiedervereinigung / vereinzelt Vereinigung): kriminelle Wiedervereinigung (taz 03.10.89, S. 8, taz 05.12.89, S. 2), die jetzt viel beschworene Wiedervereinigung (taz 07.10.89, S. 17, 18), die heißersehnte Wiedervereinigung (taz 10.10.89, S. 15), eine mögliche „Wiedervereinigung“ (taz 16.10.89, S. 17), die „,deutsche Vereinigung“ (taz 26.10.89, S. 5), die nächtliche Wiedervereinigung (taz 11.11.89, S. 11), eine mögliche deutsche Wiedervereinigung (taz 13.11.89, S. 10), eine schamlose kapitalistische Wiedervereinigung (taz 13.11.89, S. 28), die staatliche Wiedervereinigung (taz 16.11.89, S. 12-13), mediale Vereinigung (taz 16.11.89, S. 18), 
eine schleichende ,, Wiedervereinigung “ (taz 18.11.89, S. 9), eine politische wie eine ökonomische Wiedervereinigung (taz 21.11.89, S. 8), die bundesdeutsche Wiedervereinigung (taz 23.11.89, S. 8), deutsche „Vereinigung “ (taz 01.12.89, S. 1, 2), kulturelle Wiedervereinigung (taz 09.12.89, S. 29), eine ,, unkontrollierte Wiedervereinigung “ (taz 23.12 .89$, S. 4)

- Wortgruppen (adjektivisches Attribut + Nomen (Wiedervereinigung) + Genitivattribut): eine , künstlich forcierte Wiedervereinigung “ der beiden deutschen Staaten (taz 07.12.89, S. 5)

- Wortgruppen (Nomen (Wiedervereinigung) + Genitivattribut): Wiedervereinigung Deutschlands (taz 07.10.89, S. 4), Wiedervereinigung der Bundesrepublik und der DDR (taz 11.11.89, S. 4), die Wiedervereinigung der beiden Deutschländer (taz 16.11.89, S. 12-13), eine (Wieder-) Vereinigung der dritten Art (taz 01.12.89, S. 4), eine (Wieder-) Vereinigung der besonderen Art (taz 01.12.89, S. 4)

- Wortgruppen (für Wiedervereinigung steht ein anderes Nomen + Genitivattribut): die Nacht der offenen Grenze (taz 11.11.89, S. 4), Anschluß der DDR an die Bundesrepublik oder Fusion (taz 29.11.89, S. 3), die Vereinnahmung der DDR durch die BRD (taz 08.12.89, S. 21)

- Wortgruppen (adjektivisches Attribut + ein Nomen für Wiedervereinigung): die deutsch-deutsche Grenzöffnung (taz 11.11.89, S. 4), deutsch-deutscher Rausch (taz 16.11.89, S. 18), ,9. November “ (taz 21.11 .89$, S. 12)

- Wortgruppen (Wiedervereinigung + präpositionelles / situatives Attribut): Wiedervereinigung an der Bahnsteigkante (taz 06.10.89, S. 5), die ,Wiedervereinigung " von Menschen, nicht von Staaten (taz 11.11.89, S. 4), die „Wiedervereinigung von Menschen“ (taz 13.11 .89$, S. 21)

- Wortgruppen (Ausdrücke für Stimmungen und Gefühle + Wiedervereinigung als Attribut): ein Rennen in Richtung Wiedervereinigung (taz 16.10.89, S. 8), ein Pathos der Wiedervereinigung (taz 01.11.89, S. 2), der Wunsch der Deutschen nach einer Wiedervereinigung im Rahmen einer ,friedlichen und demokratischen Entwicklung " (taz 04.11.89, S. 4), der unwandelbare Wunsch nach Wiedervereinigung (taz 07.11.89, S. 8), die Wünschbarkeit oder Notwendigkeit einer sofortigen Wiedervereinigung (taz 07.11.89, S. 8), der Willen zur Wiedervereinigung (taz 09.11.89, S. 4), die Forderung nach Wiedervereinigung (taz 09.11.89, S. 4), Träume von der Wiedervereinigung 
(taz 13.11.89, S. 21), ebenso unrealistische wie gefährliche Spekulationen über eine Wiedervereinigung (taz 18.11.89, S. 1, 2), ,, Abstrakte Diskussionen über die Wiedervereinigung" (taz 20.11.89, S. 4), Bestrebungen zur Wiedervereinigung (taz 20.11.89, S. 6), Kohls ,, Gequatsche von der Wiedervereinigung “ (taz 07.12.89, S. 14), die Möglichkeit einer raschen Wiedervereinigung (taz 13.12.89, S. 5), die Forderung nach schneller ,, Wiedervereinigung “ (taz 15.12.89, S. 2)

\section{(c) Semantisches Verfahren}

- Wörter und Wortgruppen, verschiedene Strukturen, die auf wortbildenden oder syntaktischen Verfahren beruhen und jeweils metaphorisch zu verstehen sind: der Zankapfel (taz 07.10.89, S. 8), Ost-WestRendezvous (taz 13.11.89, S. 4), Zusammenbruchsphantasien (taz 17.11.89, S. 8), [Vollzug der Vereinigung ist] Kolonisierung (taz 20.11.89, S. 5), die Wiedervereinigung à la Herrhausen (taz 21.11.89, S. 8), der Wiedervereinigungsmief (taz 29.11.89, S. 2), die Wiedervereinigungswelle (taz 29.11.89, S. 3), Ost-West-Turbulenzen (taz 09.12.89, S. 1)

\section{Benennungsobjekt 3: DDR}

\section{(a) Wortbildendes Verfahren}

- Der Name ist Bestandteil eines Kompositums: DDR-Phönix (taz 26.10 .89$, S. 2)

\section{(b) Syntaktisches Verfahren}

- Enge Appositionen: Der Unruheherd DDR (taz 07.10.89, S. 4), ,Staatsgefängnis' DDR (taz 11.10.89, S. 8), Entzündungsherd DDR (taz 09.11.89, S. 18), Billiglohnland DDR (taz 18.11.89, S. 14-15), Moderneres Deutschland DDR (taz 27.11.89, S. 5), Ein Quasi-Bundesland DDR (taz 29.11.89, S. 2)

- Lockere Appositionen: die DDR der erste Friedensstaat auf deutschen (sic!) Boden (taz 01.11.89, S. 14), DDR - „Müllhalde für unsere Industrie" (taz 11.11.89, S. 5), DDR Armenhaus eines Gro $\beta$ deutschlands (taz 23.11.89, S. 2)

- Wortgruppen (adjektivisches Attribut + DDR): die gewendete DDR (taz 08.11.89, S. 8), eine entvölkerte DDR (taz 10.11.89, S. 1) 


\section{(c) Grafisches Verfahren}

- Die Ausbuchstabierung des Erstbuchstabens der Abkürzung: die deutsche DR (taz 10.11.89, S. 8)

(d) Semantisches Verfahren

- Wörter und Wortgruppen (verschiedene syntaktische Strukturen; für die Bezeichnung DDR steht jeweils ein anderes Wort oder eine Wortgruppe, die zusätzlich attribuiert werden kann): das Land des Schlimmen (taz 02.10.89, S.9), die dürftigste deutsche Kaufhalle (taz 07.10.89, S. 17, 18), Knast-Ost (taz 10.11.89, S. 8), ,,Sizilien der Bundesrepublik" (taz 11.11.89, S. 4), der bananenfreie Staat (taz 14.11.89, S. 4), zwölftes Bundesland der BRD (taz 24.11.89, S. 2), verlängerte Werkbank der Bundesrepublik (taz 30.11.89, S. 1, 2), Müllkippe Europas (taz 14.12.89, S. 3), „Billigland der BRD“ (taz 19.12.89, S. 2)

- Sprachspiel (Ausbuchstabierung der Abkürzung mit dem Austausch eines Bestandteils): Deutsche Demonstrierende Republik (taz 01.11 .89$, S. 7)

\section{Benennungsobjekt 4: BRD}

(a) Grafisches Verfahren

- Ausbuchstabierung des Letztglieds der Abkürzung: BRDeutschland (taz 01.11.89, S. 14)

(b) Wortbildendes und wortbildend-syntaktisches Verfahren

- Zusammensetzung und Attribuierung: wir ,, in diesem unserem BRDLande " (taz 23.11.89, S. 17)

- Zusammensetzung (für die Bezeichnung BRD steht ein anderes Nomen): Einwanderungsland (taz 02.10.89, S. 5)

(c) Syntaktisches Verfahren

- Enge Apposition: Konsumparadies BRD (taz 11.11.89, S. 12)

- Wortgruppe (ein Nomen für BRD + Genitivattribut): Nachfolgerin des Deutschen Reichs (taz 01.11.89, S. 5) 


\section{(d) Semantisches Verfahren}

- Wörter und Wortgruppen (verschiedene syntaktische Strukturen; für die Bezeichnung BRD steht jeweils ein anderes Wort oder eine Wortgruppe, die häufig zusätzlich attribuiert wird): das Reich des Guten (taz 02.10.89, S. 9), das Paradies (taz 02.10.89, S. 9), unsere Oase der Seligkeit (taz 02.10.89, S. 9), die Bananenrepublik (taz 14.11.89, S. 4), Der goldene Westen (taz 16.11.89, S. 18), Republik Wes (sic!) (taz 17.11.89, S. 4)

- Sprachspiel (Ausbuchstabierung der Abkürzung mit dem Austausch mehrerer Bestandteile): B(unker) R(epublik) D(rängelland) (taz 16.11.89, S. 3), BRD Bardei rädlischer Deutscher (taz 16.12.89, S. 2)

Benennungsobjekt 3 und 4: Beispiele für beide Objekte zugleich (DDR und $\mathrm{BRD} /$ Deutschland)

(a) Wortbildendes Verfahren und wortbildend-syntaktisches Verfahren

- Zusammensetzung (Verschmelzung eines Buchstabens/Wortes): BRDDR (taz 01.12.89, S. 19)

- Determinativkompositum: Großdeutschland (taz 12.12.89, S. 8), „,Großdeutschland“ (taz 14.12.89, S. 20)

- Determinativkompositum, enge Apposition: das Trauma Großdeutschland (taz 29.11.89, S. 6)

- Determinativkompositum, adjektivische Attribuierung: ein kapitalistisches und materialistisches Großkotzdeutschland (taz 21.11.89, S. 12)

(b) Syntaktisch-semantisches Verfahren

- Enge Apposition und sprachspielerische Ausbuchstabierung der Abkürzungen: „,Müllgruppe BRD/DDR“ (Bloß Rüber Damit - Dreck Dankend Retour) (taz 14.12.89, S. 3)

(c) Grafisches Verfahren

- Wortgruppe, Apposition: „,DDR + BRD Deutschland“(taz 06.12.89, S. 2). 
Benennungsobjekt 5: Bezeichnungen für ost- und westdeutsche Bürger

(a) Wortbildendes Verfahren und wortbildend-syntaktisches Verfahren

- Ableitungen (Suffigierung, Präfigierung): DDRler (taz 02.10.89, S. 2), Die Ex-DDRler (taz 02.11.89, S. 21), Ostler (taz 06.11.89, S. 24), Die Westler (taz 08.11.89, S. 1, 2), BRDlerInnen (taz 20.12.89, S. 10)

- Zusammensetzungen: Die Ostberliner (taz 02.10.89, S. 3), DDR-Bürger (taz 02.10.89, S. 5), DDR- und BRD-Bürger (taz 02.10.89, S. 5), DDR-BürgerInnen (taz 03.10.89, S. 2), Bundesbürger (taz 03.10.89, S. 8), Wir-hinter-der-Mauer (taz 07.10.89, S. 8), Das DDR-Wir (taz 07.10.89, S. 8), Die DDR-Bevölkerung (taz 13.10.89, S. 8), DDRStaatsangehörige (taz 26.10.89, S. 2), das DDR-Volk (taz 01.11.89, S. 7), Ex-DDR-Menschen (taz 07.11.89, S. 17), BundesbürgerInnen und DDR-BürgerInnen pauschal als Deutsche bezeichnet (taz 07.11.89, S. 20), Westbürger (taz 11.11.89, S. 4), Die DDR-Leute (taz 11.11.89, S. 9), Die Noch-DDR-Bürger (taz 11.11.89, S. 25)

- Kurzwörter: Ossis (taz 11.11.89, S. 7), Wessis (taz 17.11.89, S. 4), Bundis (taz 21.11.89, S. 24), Zonis (taz 21.11.89, S. 24)

- Kurzwort und Attribuierung: Die armen Zonis (taz 27.11.89, S. 17)

- Präfigierung und Attribuierung: [die] neuen Mitbürger (taz 06.10.89, S. 5), unsere netten Neubürger (taz 10.10.89, S. 20), die jungen, guterhaltenen und tatendurstigen Neubürger (taz 11.10.89, S. 8)

\section{(b) Syntaktisches Verfahren}

- Wortgruppen (Nomen + Attribute; adjektivische, genitivische, situative Attribute, possessive Determinativattribute, Apposition): Bürger der DDR (taz 03.10.89, S. 8), die ,LLandsleute von drüben “ im ,, freien Teil Deutschlands “ (taz 11.10.89, S. 8), Bürger und Bürgerinnen der DDR (taz 18.10.89, S. 2-3), Die ewig nörgelnde DDR-Bevölkerung (taz 01.11.89, S. 12), das Volk der DDR, die Weggeher und Dableiber (taz 16.11.89, S. 12-13), die Deutschen in Ost und West (taz 29.11.89, S. 3), unsere Brüder in der DDR (taz 20.12.89, S. 10)

- Wortgruppe (Paarformel): Dableiber und Ausreiser (taz 08.11.89, S. 8)

(c) Semantisches Verfahren

- Wörter und Wortgruppen (verschiedene Strukturen; Metaphern): Reaktionäre Ostlandritter (taz 03.10.89, S. 8), Die ,ewig Gestrigen “ (taz 04.10.89, S. 17), [Macht eure Revolution zu Hause,] TouristInnen! 
(taz 13.11.89, S. 5), Die Kinder von Marx und Coca Cola (taz 14.11.89, S. 4), In Unmündigkeit gehaltene Kinder eines Erziehungsheims hinter Stacheldraht (taz 18.11.89, S. 14-15)

\section{Benennungsmotive: ein Kommentar}

Im Folgenden werde ich einige Bemerkungen zu der vorangehenden Übersicht geben und Schlussfolgerungen für eine sprachliche Konstruktion der gesellschaftlichen und politischen Wirklichkeit ziehen.

Das Bildungsverfahren steht im Zusammenhang mit den von Sprechenden angestrebten Benennungsmotiven, die in den Bezeichnungen zum Ausdruck gebracht werden. Sollen bestimmte Merkmale von Objekten oder die Einstellung zu ihnen signalisiert werden, können sie in direkter Darstellungsweise durch Attribute indiziert werden. Hier eröffnen sich die folgenden Möglichkeiten: Erstens, die Sprechenden können den Sachverhalt lediglich mitteilen - im Sinne einer informativen Formulierungsweise neutral und sachbetont (Bsp. Wiedervereinigung der Bundesrepublik und der DDR, die staatliche Wiedervereinigung) oder sie können eine wertende Stellungnahme ausdrücken, entweder positiv (Bsp. eine (Wieder-)Vereinigung der besonderen Art) oder negativ (Bsp. kriminelle Wiedervereinigung). Zweitens, sie können die Benennungen so gestalten, dass sie ein emotionales Empfinden, eine Erregung widerspiegeln bzw. sie auslösen (sollen). Auf diese Weise können den Rezipierenden die eigenen Zweifel kundgetan (Bsp. eine mögliche deutsche Wiedervereinigung), Erwartungen vermittelt (Bsp. die heißersehnte Wiedervereinigung) oder eine Identifikation mit ihnen signalisiert werden (als werde im Namen einer Gruppe gesprochen, wie etwa in [jetzt, 1989, haben wir] unsere Oktoberrevolution, unsere Brüder in der DDR). Eignen sich die lexikalisierten Ausdrücke nicht dazu, den Benennungszweck zu erfüllen, fängt die kreative Phase in der Formulierungsweise an. Die Sprechenden bilden okkasionelle Bezeichnungen (Bsp. die Wiedervereinigung à la Herrhausen, die Krenz-Wende, Wir-hinterder-Mauer). In vielen Fällen handelt es sich dabei um Lexeme, denen man im alltäglichen Sprachgebrauch begegnet, die aber in neuen Kontexten oder originellen Zusammenhängen und als neue Wortverbindungen entsprechend der jeweiligen Kommunikationssituation eingesetzt werden (Bsp. ,Stunde-Null “Rhetorik). Die situations-okkasionellen, oft bildhaften Benennungsvarianten können auch besser zur Veranschaulichung komplexer und komplizierter Sachverhalte dienen, wenn zu in der Alltagssprache bereits etablierten Formu- 
lierungen gegriffen wird. In kreativen Ausprägungen ist für das korrekte Verstehen ausschlaggebend, dass die Rezipierenden über ein entsprechendes thematisches und kontextuelles Hintergrundwissen verfügen. ${ }^{7}$

In den angeführten Beispielgruppen finden sich Bezeichnungsketten (oder Bezeichnungsreihen), die durch ein festes Lexem verbunden sind. ${ }^{8}$ So wird dasselbe Benennungsobjekt etwa durch das Nomen Revolution erfasst und durch verschiedene Attribute erweitert (klug, sanft, demokratisch, erfolgreich, gewaltfrei, friedlich etc.) oder es ist Bestandteil einer Zusammensetzung (Novemberrevolution des Jahres ..., Oktoberrevolution). Durch eine gewisse Musterhaftigkeit der Bezeichnungsreihen haben Sprechende die Möglichkeit, den Lesenden entsprechend der jeweiligen Situation jeweils spezifische Sichtweisen des Benannten darzustellen und zugleich Wertungen abzugeben. Da die Varianten verschiedene Objektmerkmale fixieren, können sie bei den Rezipierenden breite Assoziationspotenziale eröffnen und verschieden konnotativ wirken. Wiedervereinigung kann in ein Begriffsfeld eingeordnet werden, dass zu umreißen ist mit z. B. Vertrauen, Hoffnung, Entscheidungsfreiheit, Freiheit von Meinungen, Verbreitung des persönlichen Handlungsspielraums, Mitsprache (ermöglichen), Rücksicht (nehmen und erfahren), Umkehr der Verhältnisse (im positiven Sinn), aber auch mit Angst / Ängste, Gewalt, Unsicherheit, Zwang (sich anpassen zu müssen), Umkehr der Verhältnisse (im negativen Sinn) etc. Durch die Vernetzung mit verschiedenen Werten und ihre sprachliche Realisierung (Mehrfachbenennung) nimmt das Wort häufig den Rang eines Schlüsselwortes ein. ${ }^{9}$

Viele Beispiele im Korpus stehen in Anführungszeichen, die einige Interpretationsmöglichkeiten eröffnen. Durch die Setzung von Anführungszeichen

7 Verstehensrelevantes Wissen war nicht Thema der Diskussion. $\mathrm{Zu}$ einem linguistischen Modell des Textverstehens bzw. der Textinterpretation vgl. z. B. Busse (1992). Ich verwende hier den Terminus Bezeichnungskette etwas anders als ihn z. B. SCHIPPAN (1992:194) definiert. Mit Bezeichnungsketten (auch nominativen Ketten) meint sie allgemein eine Reihe von verschiedenen Benennungseinheiten, die sich im konkreten Text auf das gleiche Objekt beziehen und referenzielle Beziehungen, den inhaltlichen Zusammenhang und semantische Verflechtungen im Text herstellen.

In diesem Kontext verstehe ich Schlüsselwörter nach LIEBERT eben als solche, die „das Selbstverständnis und die Ideale einer Gruppe oder einer ganzen Epoche ausdrücken können. Sie sollen diskursbestimmend sein. Die kontextuelle und konnotative Bedeutung soll dominant sein. Sie sollen umstritten sein. Sie sollen eine große Bedeutungsvielfalt aufweisen." (LIEBERT 2003:59f.) 
kann signalisiert werden, dass die Verfassenden lediglich andere zitieren und die Verantwortung für das Gesagte diesen überlassen (es handelt sich hierbei um die sog. referierende Funktion von Anführungszeichen). Durch die Wahl von Anführungszeichen können Sprechende auch eine Eigenschaft des Objekts speziell grafisch markieren, um das für sie Wichtige am Sachverhalt zu betonen, um die Aufmerksamkeit der Lesenden dadurch zu steuern oder um zu signalisieren, dass das Gesagte nicht ernst (spöttisch, umgekehrt o. ä.) oder nicht wortwörtlich (sondern metaphorisch) zu verstehen ist.

Abschließend kehre ich noch einmal zu den Okkasionalismen zurück. Es gibt im Belegkorpus einige Benennungen, die auf Sprachspielen beruhen. Es sind u. a. die sprachspielerischen Ausbuchstabierungen und Anspielungen, die offensichtlich aus der aktuellen politisch-gesellschaftlichen Situation resultieren und als Ausdruck der subjektiven Einstellung der Sprechenden zu betrachten sind. Sie dienen vielen Zwecken. So üben sie z. B. Gesellschaftskritik (Bsp. „Müllgruppe BRD/DDR“ Bloß Rüber Damit - Dreck Dankend Retour), weisen verschiedene Gefühle (Angst, Verzweiflung, Freude) angesichts des politischgesellschaftlichen Alten und Neuen auf (Bsp. die friedliche Revolution vom November 1989), sie dienen zugleich auch der Erzielung einer scherzhaften Wirkung (Bsp. BRD Bardei rädlischer Deutscher). Die wir-Formen in solchen Äußerungen, auch in possessiver Formulierungsweise (Bsp. [jetzt, 1989, haben wir] unsere Oktoberrevolution), bezwecken, Gleichgesinnte in Hinsicht auf die Beurteilung der gesellschaftlichen Beziehungen zu finden und $\mathrm{zu}$ verbinden. Darüber hinaus lässt sich eine Fülle von metaphorischen Benennungen beobachten (Bsp. die Bananenrepublik, das Paradies), deren Bildung sich durch das Streben nach ausdrucksstärkeren und expressiven Konkurrenzformen erklärt, von denen Sprechende Gebrauch machen, um emotionale Involviertheit zu markieren. Sie sind zudem erfolgreicher Rezeptionsanreiz, ziehen die Aufmerksamkeit der Rezipierenden auf sich und werden möglicherweise dazu verwendet, bestimmte Wertungen und Gefühlszustände zu aktivieren oder zu provozieren.

\section{Schlussbemerkungen}

Anhand der oben angeführten Beispiele wird ersichtlich, dass bei Benennungsausdrücken folgende Kriterien zu berücksichtigen sind: (a) das Benennungsverfahren, (b) der existierende Benennungsvorrat für Objekte der außersprachlichen Realität (d. h. die vorhandenen Erstbenennungen, prinzipiell Standardbenennungen), (c) die Einstellung der Sprechenden zum benannten Objekt 
(realisiert in Zweitbenennungen und situations-okkasionellen Benennungen, die subjektive Wahrnehmungsresultate vom gleichen Objekt repräsentieren) und (d) die angestrebten Reaktionen bei den Rezipierenden (sie entsprechen den Benennungsmotiven seitens der Sprechenden).

Die genannten Kriterien bedingen einander. Die Benennungsverfahren hängen mit den entsprechenden morphosyntaktischen Strukturen von Benennungsausdrücken zusammen, die in der Beispielübersicht nach dem wortbildenden, dem syntaktischen und dem semantischen Verfahren geordnet sind. Die analysierten Benennungsobjekte charakterisieren sich durch strukturell verschiedenartige und durch mehrfache Benennungen. Man begegnet Erstbenennungen, Erstbenennungen mit Attribuierungen, Zweitbenennungen und situations-okkasionellen Benennungen. Viele Benennungen sind keine festgeprägten Standardbenennungen. Die Wahl des Benennungsverfahrens hängt davon $a b$, wie viele lexikalisierte Ausdrücke, die sich auf ein bestimmtes Benennungsobjekt beziehen, den Sprechenden zur Verfügung stehen. Neue und okkasionelle referenzidentische Ausdrücke fungieren als Benennungsvarianten zu einem mit neutraler Standardbenennung in den Text eingeführten Benennungsobjekt. Sie lassen mehr Eigenschaften des Objekts erkennen und sorgen für die Herstellung eines inhaltlichen Zusammenhangs sowie semantischer Verflechtungen im Text (die textinterne Referenz; man berücksichtige auch eine eventuelle stilistische Merkmalhaftigkeit der Ausdrücke). Ausdrucksstärkere Konkurrenzformen für Standardbenennungen, die Resultat subjektiver (oder veränderter) Benennungsmotive sind, signalisieren die spezifische Sichtweise des Benennenden. Zugleich ermöglichen sie es in einem höheren Maße, die Eigenschaften des Benennungsobjekts zu exponieren, zu verschleiern oder zu parodieren. Solche Benennungen erklären sich vor allem aus der gefühlsmäßigen Beteiligung der Sprechenden an der Wahrnehmung der außersprachlichen Realität (textexterne Referenz). Auf der anderen Seite werden sie möglicherweise auch aus dem Bedürfnis heraus geschaffen, bestimmte Reaktionen der Rezipierenden $\mathrm{zu}$ antizipieren. Abgesehen von der linguistischen Betrachtung ist hier abschließend festzuhalten, dass die Sprache überzeugend und fast unfehlbar zeigte, was Realität wurde. Sie hat auf jeden Fall sensibel auf politische und gesellschaftliche Veränderungen reagiert. 
Zu Bildungsverfahren und Strukturen von Benennungsausdrücken. Eine Analyse...

\section{Literatur}

BRINKER, KLAUS ('2005): Linguistische Textanalyse. Eine Einführung in Grundbegriffe und Methoden. Berlin.

BusSE, DiETRICH (1992): Textinterpretation. Sprachtheoretische Grundlagen einer explikativen Semantik. Opladen.

FEINE, ANGELIKA (1997): Mit Spritfressern in die Klimakatastrophe? Betrachtungen zu Mehrfachbenennungen in publizistischen Texten. In: KESSLER, CHRISTINE / SOMMERFELDT, KARL-ERNST (eds.): Sprachsystem - Text - Stil. Festschrift für Georg Michel und Günter Starke zum 70. Geburtstag. Frankfurt a. M., 61-73.

- (1999): Zur Benennungsmotivation beim Gebrauch von Nominationsvarianten. In: DöRING, BRIGITTE / FEINE. ANGELIKA / SCHELlENBERG, WiLHELM (eds.): Über Sprachhandeln im Spannungsfeld von Reflektieren und Benennen. Frankfurt a. M., 73-88.

- (2000): Benennungsausdrücke: Bildungsverfahren und Strukturen. In: FEINE, ANGELIKA / ŻydEK-BEDNARCZUK, URSZUla (eds): Beiträge zur Nomination im Deutschen und Polnischen. Katowice, 9-24.

FleISCHER, WolfGANG (1989): Nomination und unfeste nominative Ketten. In: FleISCHER, WOLFGANG / GROSSE, RUdOLF / LERCHNER, GOTTHARD (eds.): Beiträge zur Erforschung der deutschen Sprache 9. Leipzig, 13-27.

GŁADYSZ, MAREK (2006): Morphosyntaktische und onomasiologische Struktur von Nominationseinheiten. In: Cirko, LesŁAw / Grimberg, MARTIN (eds.): Phänomene im syntaktisch-semantischen Grenzbereich. Materialien der internationalen Linguistenkonferenz Karpacz 27.-29.09.2004. Dresden / Wrocław, 103-116.

- (2007): Kreativität und Sprachlust bei der Mehrfachbenennung. In: CIRKO, LeSŁAw / Grimberg, MARTIN (eds.): Sprachlust - Norm - Kreativität. Materialien der internationalen Linguistenkonferenz Karpacz 12.-14.09.2005. Dresden / Wrocław, 83-93.

GołęBIOWsKi, AdAm / ENGEL, UlRich (2014): Sachen charakterisieren. In: EngEL, Ulrich / BŁACHut, Edyta / GoŁęBIOWSKi, AdAm / JuRAsZ, AlinA (eds.): Über Sachen reden. Sprechen im deutsch-polnischen Kontrast. Hamburg, 67-95.

Grice, Herbert Paul (1957): Meaning. In: The Philosophical Review 66: 377-388. [Ins Deutsche übersetzt von Georg MegGle als Intendieren, Meinen, Bedeuten. In: MegGle, GEORG (ed.) (1979): Handlung, Kommunikation, Bedeutung. Frankfurt a. M., 2-15.]

- (1969): Utterer's Meaning and Intentions. In: The Philosophical Review 78: 147-177. [Ins Deutsche übersetzt von GEORG MEgGLE als Sprecher-Bedeutung und Intentionen. In: Meggle, Georg (ed.) (1979): Handlung, Kommunikation, Bedeutung. Frankfurt a. M., 16-51.]

Hellmann, MANFRED W. (2006): Wörter in Texten der Wendezeit. Ein Wörterbuch zum „,Wendekorpus“ des IDS. Mai 1989 bis Ende 1990. Unter Mitwirkung von PANTELIS Nikitopoulos und Christoph MelK. CD-ROM mit Begleitband. Mannheim. 
Herberg, Dieter / StefFens, Doris / Tellenbach, Elke (1997): Schlüsselwörter der Wendezeit. Wörter-Buch zum öffentlichen Sprachgebrauch 1989/90. Berlin / New York (= Schriften des Instituts für Deutsche Sprache 6).

Keller, Rudi (1995): Zeichentheorie. Zu einer Theorie semiotischen Wissens. Tübingen / Basel.

Knobloch, Clemens (1996): Nomination: Anatomie eines Begriffs. In: KnOBloch, Clemens / Schaeder, Burkhard (eds.): Nomination - fachsprachlich und gemeinsprachlich. Opladen, 21-54.

Knobloch, Clemens / Schaeder, Burkhard (1996): Nomination - fachsprachlich und gemeinsprachlich. Ein Vorwort. In: KNOBLOCH, ClEMENS / SCHAEDER, BURKHARD (eds.): Nomination - fachsprachlich und gemeinsprachlich. Opladen, 7-19.

LIEBERT, WOLF-ANDREAS (2003): Zu einem genetischen Konzept von Schlüsselwörtern. In: Zeitschrift für Angewandte Linguistik 38:57-75.

LÜGER, HeINZ-Helmut (1995): Pressesprache. Tübingen.

Polenz, Peter von (1985): Deutsche Satzsemantik. Grundbegriffe des Zwischen-denZeilen-Lesens. Berlin / New York.

POSNER, ROLAND (1992): Believing, causing, intending: The basis for a hierarchy of sign concepts in the reconstruction of communication. In: JORNA, RENÉ J. / VAN Heusden, Barend / Posner, Roland (eds.): Sign, Search and Communication: Semiotic Aspects of Artificial Intelligence. Berlin / New York, 215-270.

SCHIPPAN, THEA (1992): Lexikologie der deutschen Gegenwartssprache. Tübingen.

Wagner, KLAus R. (2001): Pragmatik der deutschen Sprache. Frankfurt a. M. 